\title{
Deterioration of Cerebral Oxygenation by Aortic Arch Calcification Progression in Patients Undergoing Hemodialysis: A Cross-Sectional Study
}

\author{
Kiyonori Ito, ${ }^{1}$ Susumu Ookawara, ${ }^{1}$ Tomohisa Okochi, ${ }^{2}$ \\ Yuichiro Ueda, ${ }^{1}$ Masaya Kofuji, ${ }^{3}$ Hideyuki Hayasaka, ${ }^{3}$ Takayuki Uchida, ${ }^{3}$ \\ Haruhisa Miyazawa, ${ }^{1}$ Katsunori Yanai, ${ }^{1}$ Hiroki Ishii, ${ }^{1}$ Taisuke Kitano, ${ }^{1}$ \\ Mitsutoshi Shindo, ${ }^{1}$ Keiji Hirai, ${ }^{1}$ Yoshio Kaku, ${ }^{1}$ Taro Hoshino, ${ }^{1}$ Osamu Tanaka, ${ }^{2}$ \\ Kaoru Tabei, ${ }^{4}$ and Yoshiyuki Morishita ${ }^{1}$ \\ ${ }^{1}$ Division of Nephrology, Department of Integrated Medicine, Saitama Medical Center, Jichi Medical University, Saitama, Japan \\ ${ }^{2}$ Division of Radiology, Department of Integrated Medicine, Saitama Medical Center, Jichi Medical University, Saitama, Japan \\ ${ }^{3}$ Department of Clinical Engineering, Saitama Medical Center, Jichi Medical University, Saitama, Japan \\ ${ }^{4}$ Minami-Uonuma City Hospital, Niigata, Japan
}

Correspondence should be addressed to Susumu Ookawara; su-ooka@hb.tp1.jp

Received 14 May 2017; Revised 5 August 2017; Accepted 24 August 2017; Published 3 October 2017

Academic Editor: John Maesaka

Copyright (c) 2017 Kiyonori Ito et al. This is an open access article distributed under the Creative Commons Attribution License, which permits unrestricted use, distribution, and reproduction in any medium, provided the original work is properly cited.

\begin{abstract}
Background. Near-infrared spectroscopy revealed that the regional saturation of oxygen $\left(\mathrm{rSO}_{2}\right)$ in cerebral tissue is lower in hemodialysis (HD) patients than in healthy subjects. However, no study has examined the changes in cerebral oxygenation by aortic arch calcification (AAC) progression in HD patients. Methods. A total of $104 \mathrm{HD}$ patients were divided into four groups by AAC grade determined using chest radiography: 23 patients at grade 0,24 at grade 1, 30 at grade 2, and 27 at grade 3 . Differences in clinical parameters, including cerebral $\mathrm{rSO}_{2}$, among AAC grades were investigated and atherosclerotic parameters affecting cerebral $\mathrm{rSO}_{2}$ values were identified. Results. Cerebral $\mathrm{rSO}_{2}$ significantly decreased as AAC progressed (AAC grade 3 versus grade $0, p<0.01$ versus grade $1, p<0.05)$. Multivariate logistic regression analysis was performed using parameters with $p$ values $<$ 0.20 in univariate analysis between cerebral $\mathrm{rSO}_{2}$ values less than the mean and atherosclerotic parameters. AAC grades 2 and 3 , serum phosphate level, and history of smoking were factors associated with the cerebral $\mathrm{rSO}_{2}$ decrease. Conclusions. Cerebral $\mathrm{rSO}_{2}$ significantly decreased as AAC progressed and was independently associated with higher AAC grade, serum phosphate level, and history of smoking.
\end{abstract}

\section{Introduction}

According to a 2012 year-end report, cerebrovascular disease is a main cause of death in patients receiving hemodialysis (HD) in Japan [1]. A lower estimated glomerular filtration rate is associated with lower cerebral blood flow [2], and patients with chronic kidney disease or who were receiving HD tend to develop cerebral infarction with high frequency even if they were asymptomatic $[3,4]$. Therefore, it is important to recognize that patients with renal impairment may have vascular disorders, including ischemic stroke. Regarding the evaluation of vascular disorders, aortic arch calcification
(AAC) using chest radiography has been a concern in clinical settings $[5,6]$. To date, AAC was reportedly associated with cerebral ischemic disorder [7], in addition to cardiovascular disease [8].

Near-infrared spectroscopy (NIRS) has recently been used in various clinical settings to measure the regional saturation of oxygen $\left(\mathrm{rSO}_{2}\right)$, a real-time marker of tissue oxygenation [9-14]. Cerebral $\mathrm{rSO}_{2}$ values were reportedly significantly lower in patients receiving $\mathrm{HD}$ than in healthy controls $[15,16]$. Furthermore, these values were associated with $\mathrm{pH}, \mathrm{HD}$ duration, serum albumin concentration, and the presence of diabetes mellitus [16]. However, no studies 
have examined the correlation between cerebral oxygenation and vascular disorders represented by AAC in HD patients. Therefore, here we aimed to clarify the associations between AAC and clinical parameters, including cerebral $\mathrm{rSO}_{2}$ values, in patients receiving $\mathrm{HD}$.

\section{Materials and Methods}

We included patients receiving $\mathrm{HD}$ who met the following criteria: (1) with ESRD receiving intermittent HD, (2) AAC grade confirmed by chest radiography, and (3) presence of arteriovenous fistula as vascular access for HD. The exclusion criteria included any uncontrolled patients' condition: congestive heart failure with pleural effusion, acute cerebrovascular disease, obvious disturbing condition including cognitive impairment, and lung disease with shortness of breath at rest or high concentrated oxygen administration.

One hundred and four patients receiving $\mathrm{HD}$ were included (80 men and 24 women; mean age, $67.4 \pm 9.6$ years; mean HD duration, $4.7 \pm 6.7$ years). The causes of chronic renal failure were diabetes mellitus (51 patients), chronic glomerulonephritis (23 patients), nephrosclerosis (15 patients), and other (15 patients). Each patient was receiving maintenance HD two or three times a week with each HD session lasting 3 or 4 hours. The patients' general characteristics are summarized in Table 1 . Written informed consent was obtained from all participants and this study was approved by the Institutional Review Board of Saitama Medical Center, Jichi Medical University, Japan (RIN 14-24), and conformed to the provisions of the Declaration of Helsinki (as revised in Tokyo in 2004).

\subsection{Data Collection}

2.1.1. Patients' Baseline Characteristics and Clinical Laboratory Measurement. We collected the patients' baseline characteristics and other relevant data from their medical charts. The primary disease causing the dialysis requirement as well as any history of ischemic heart disease (IHD), cerebrovascular disease, or smoking, were recorded on the basis of medical records in our hospital.

Blood pressure (BP) and heart rate were measured with patients in the supine position before the HD session at the same time as the cerebral $\mathrm{rSO}_{2}$ measurement. Each patient's body mass index was calculated as weight (kg) divided by the square of height $\left(\mathrm{m}^{2}\right)$ by each patient's dry weight. Blood samples were obtained at ambient temperature from the arteriovenous fistula of each patient prior to HD.

Chest radiography was performed within 1 month of cerebral $\mathrm{rSO}_{2}$ measurement, and the AAC grade was determined by two or more radiologists, as recommended in previous reports $[5,6]$. We then examined the associations between AAC grade and clinical parameters, including cerebral $\mathrm{rSO}_{2}$ value, in each patient.

2.1.2. Cerebral Oxygenation Monitoring. Cerebral $\mathrm{rSO}_{2}$ was monitored at the forehead using an INVOS 5100C saturation monitor (Covidien Japan, Tokyo, Japan) that utilizes NIRS technology. This instrument uses a light-emitting diode, which transmits near-infrared light at two wavelengths (735 $\mathrm{nm}$ and $810 \mathrm{~nm}$ ), and two silicon photodiodes that act as light detectors to measure oxygenated $\mathrm{Hb}$ and deoxygenated $\mathrm{Hb}$. The ratio of oxygenated $\mathrm{Hb}$ to total $\mathrm{Hb}$ (oxygenated $\mathrm{Hb}+$ deoxygenated $\mathrm{Hb}$ ) signal strength, which is the corresponding percentage, is read as a single numerical value that represents the $\mathrm{rSO}_{2}[17,18]$.

All data obtained using this instrument were immediately and automatically stored in sequence. The interobserver variance for this instrument, that is, reproducibility of the $\mathrm{rSO}_{2}$ measurement, is acceptable as previously reported [19-21]. Therefore, cerebral $\mathrm{rSO}_{2}$ is considered reliable for estimating the actual cerebral oxygenation. Furthermore, the light paths leading from the emitter to the different detectors share a common part: the $30 \mathrm{~mm}$ detector assesses superficial tissue, while the $40 \mathrm{~mm}$ detector is used to assess deep tissue. By analyzing the differential signals collected by the different detectors, the current data for cerebral $\mathrm{rSO}_{2}$ values were supposed to be obtained in deep tissue $20-30 \mathrm{~mm}$ from the body's surface $[22,23]$. These measurements were performed at every 6-second interval during cerebral oxygenation monitoring.

Prior to undergoing $\mathrm{HD}$, the included patients rested in the supine position for at least 10 minutes to reduce the influence of postural change. An $\mathrm{rSO}_{2}$ measurement sensor was attached to the patient's forehead for measurement in the resting state. Thereafter, $\mathrm{rSO}_{2}$ was measured for $5 \mathrm{~min}$ before $\mathrm{HD}$, and we evaluated the mean $\mathrm{rSO}_{2}$ for $5 \mathrm{~min}$, as a marker of cerebral oxygenation, in each patient.

2.1.3. Inclusion of Atherosclerotic Parameters. In this study, we examined the relationship between cerebral $\mathrm{rSO}_{2}$ values and atherosclerotic parameters in addition to the severity of AAC grade, using univariate and multivariate logistic regression analysis. Atherosclerosis/arteriosclerosis was reportedly increased with cardiovascular risk factors, including advanced aging, smoking, hypertension, diabetes mellitus, dyslipidemia, abnormal calcium/phosphate metabolism, and inflammation $[24,25]$. Therefore, we included several clinical parameters as atherosclerotic parameters in univariate and multivariate logistic regression analysis as follows: age, smoking, systolic and diastolic BP, serum calcium and serum phosphate concentration, high density lipoprotein cholesterol, low density lipoprotein cholesterol, triglyceride, blood glucose, hemoglobin Alc, C-reactive protein levels, and severity of AAC grade.

2.2. Statistical Analysis. Data are expressed as mean \pm standard deviation. An unpaired Student's $t$-test was used to compare the two groups. One-way analysis of variance with Tukey's test was used to evaluate the significance among the four groups that were divided by AAC grade. A multivariate logistic regression analysis was performed to identify the atherosclerotic factors associated with a cerebral $\mathrm{rSO}_{2}$ value less than the mean using stepwise procedure. In this study, the cut-off value for cerebral $\mathrm{rSO}_{2}$ was $48.6 \%$. The atherosclerotic parameters with a $p$ value of 0.20 in univariate analysis were included in a stepwise manner. All analyses were 
TABLE 1: Baseline characteristics by AAC grade.

\begin{tabular}{|c|c|c|c|c|}
\hline AAC grade & Grade 0 & Grade 1 & Grade 2 & Grade 3 \\
\hline$N$ & 23 & 24 & 30 & 27 \\
\hline Sex (men), $n(\%)$ & $18(78)$ & $20(83)$ & $21(70)$ & $21(78)$ \\
\hline Age, years & $64 \pm 11$ & $64 \pm 10$ & $71 \pm 8^{c}$ & $70 \pm 7$ \\
\hline HD duration, years & $2.6 \pm 5.7$ & $5.0 \pm 7.6$ & $4.3 \pm 7.5$ & $6.7 \pm 5.5$ \\
\hline \multicolumn{5}{|l|}{ Primary disease, $n(\%)$} \\
\hline Diabetes mellitus & $11(48)$ & $7(29)$ & $20(70)^{\mathrm{c}}$ & $13(48)$ \\
\hline Nephrosclerosis & $2(9)$ & $4(17)$ & $4(12)$ & $5(19)$ \\
\hline Chronic glomerulonephritis & $8(34)$ & $8(33)$ & $4(12)$ & $3(11)$ \\
\hline Others & $2(9)$ & $5(21)$ & $2(6)$ & $6(22)$ \\
\hline \multicolumn{5}{|l|}{ Medical history, $n(\%)$} \\
\hline Ischemic heart disease & $3(13)$ & $10(42)$ & $8(28)$ & $20(74)^{\mathrm{bd}}$ \\
\hline Cerebral infarction & $4(17)$ & $8(33)$ & $4(14)$ & $4(15)$ \\
\hline Smoking & $12(52)$ & $22(92)^{\mathrm{a}}$ & $17(61)$ & $20(74)$ \\
\hline Body mass index, $\mathrm{kg} / \mathrm{m}^{2}$ & $22 \pm 4$ & $23 \pm 3$ & $22 \pm 3$ & $23 \pm 3$ \\
\hline Systolic BP, mmHg & $145 \pm 23$ & $139 \pm 18$ & $150 \pm 20$ & $137 \pm 23$ \\
\hline Diastolic BP, mmHg & $79 \pm 15$ & $78 \pm 13$ & $73 \pm 13$ & $69 \pm 15$ \\
\hline Pulse pressure, $\mathrm{mmHg}$ & $66 \pm 16$ & $62 \pm 18$ & $77 \pm 20^{c}$ & $69 \pm 14$ \\
\hline Heart rate, per min & $76 \pm 11$ & $75 \pm 15$ & $71 \pm 16$ & $73 \pm 12$ \\
\hline Oxygen saturation, $\%$ & $96 \pm 1$ & $95 \pm 2$ & $94 \pm 5$ & $95 \pm 3$ \\
\hline \multicolumn{5}{|l|}{ Medication, $n(\%)$} \\
\hline RAS blocker (ACEI and/or ARB) & $12(52)$ & $15(63)$ & $17(57)$ & $14(52)$ \\
\hline Calcium channel blocker & $14(61)$ & $15(63)$ & $22(73)$ & $15(56)$ \\
\hline Beta blocker & $8(35)$ & $12(50)$ & $19(63)$ & $15(56)$ \\
\hline Vitamin D analog & $11(48)$ & $10(42)$ & $13(43)$ & $11(41)$ \\
\hline Phosphate binder & $15(65)$ & $13(54)$ & $19(63)$ & $18(67)$ \\
\hline Calcium-containing phosphate binder & 14 & 10 & 15 & 13 \\
\hline Non-calcium-containing phosphate binder & 4 & 7 & 10 & 13 \\
\hline Cinacalcet & $1(4)$ & $1(4)$ & $3(10)$ & $4(15)$ \\
\hline Statin & $5(22)$ & $8(33)$ & $10(33)$ & $12(44)$ \\
\hline Antiplatelet agents & $8(35)$ & $11(46)$ & $13(43)$ & $19(70)$ \\
\hline Erythropoiesis stimulating agent & $21(91)$ & $22(92)$ & $30(100)$ & $24(89)$ \\
\hline Albumin, g/dL & $3.3 \pm 0.6$ & $3.2 \pm 0.5$ & $3.1 \pm 0.5$ & $2.9 \pm 0.6^{\mathrm{a}}$ \\
\hline Serum sodium, mEq/L & $138 \pm 4$ & $137 \pm 5$ & $137 \pm 3$ & $137 \pm 4$ \\
\hline Serum potassium, mEq/L & $4.4 \pm 0.8$ & $4.1 \pm 0.8$ & $4.2 \pm 0.8$ & $4.1 \pm 0.5$ \\
\hline Serum calcium, mg/dL & $8.3 \pm 0.7$ & $8.9 \pm 1.1$ & $8.5 \pm 1.1$ & $8.8 \pm 0.9$ \\
\hline Serum phosphate, $\mathrm{mg} / \mathrm{dL}$ & $4.8 \pm 1.2$ & $4.4 \pm 1.5$ & $4.8 \pm 1.3$ & $4.4 \pm 1.3$ \\
\hline BUN, mg/dL & $60 \pm 14$ & $55 \pm 26$ & $52 \pm 20$ & $51 \pm 17$ \\
\hline $\mathrm{Cr}, \mathrm{mg} / \mathrm{dL}$ & $9.1 \pm 2.2$ & $8.3 \pm 2.2$ & $8.5 \pm 2.4$ & $8.2 \pm 2.2$ \\
\hline $\mathrm{Hb}, \mathrm{g} / \mathrm{dL}$ & $9.9 \pm 1.3$ & $9.9 \pm 1.6$ & $9.4 \pm 1.5$ & $9.3 \pm 1.5$ \\
\hline Blood glucose, mg/dL & $159 \pm 73$ & $149 \pm 74$ & $133 \pm 37$ & $152 \pm 48$ \\
\hline HbAlc, \% & $5.5 \pm 1.0$ & $5.5 \pm 0.8$ & $5.8 \pm 1.0$ & $5.7 \pm 0.8$ \\
\hline Total cholesterol, mg/dL & $156 \pm 32$ & $148 \pm 39$ & $151 \pm 40$ & $149 \pm 32$ \\
\hline HDL cholesterol, mg/dL & $47 \pm 20$ & $43 \pm 16$ & $45 \pm 14$ & $36 \pm 14$ \\
\hline Non-HDL cholesterol, mg/dL & $109 \pm 24$ & $107 \pm 29$ & $105 \pm 36$ & $114 \pm 29$ \\
\hline LDL cholesterol, mg/dL & $84 \pm 23$ & $77 \pm 26$ & $80 \pm 31$ & $88 \pm 28$ \\
\hline Triglyceride, mg/dL & $96 \pm 45$ & $111 \pm 33$ & $100 \pm 54$ & $117 \pm 57$ \\
\hline C-reactive protein, $\mathrm{mg} / \mathrm{dL}$ & $0.7 \pm 1.4$ & $3.4 \pm 8.8$ & $1.2 \pm 1.6$ & $3.2 \pm 4.9$ \\
\hline Cerebral $\mathrm{rSO}_{2}$ value, $\%$ & $53.7 \pm 8.3$ & $50.1 \pm 10.1$ & $48.3 \pm 8.6$ & $43.2 \pm 8.8^{\mathrm{bc}}$ \\
\hline
\end{tabular}

AAC, aortic arch calcification; HD, hemodialysis; BP, blood pressure; RAS, renin-angiotensin system; ACEI, angiotensin-converting enzyme inhibitor; ARB, angiotensin II receptor blocker; BUN, blood urea nitrogen; Cr, creatinine; Hb, hemoglobin; HbAlc, hemoglobin Alc; HDL, high density lipoprotein; LDL, low density lipoprotein; $\mathrm{rSO}_{2}$, regional saturation of oxygen. ${ }^{\mathrm{a} S i g n i f i c a n t}$ versus the AAC grade 0 group $(p<0.05)$. ${ }^{\mathrm{b}}$ Significant versus the AAC grade 0 group $(p<0.01) .{ }^{c}$ Significant versus the AAC grade 1 group $(p<0.05) .{ }^{\mathrm{d}}$ Significant versus the AAC grade 2 group $(p<0.01)$. 
TABLE 2: Multivariate regression analysis for cerebral $\mathrm{rSO}_{2}$ value.

\begin{tabular}{lccc}
\hline & $\begin{array}{c}\text { Univariate analysis } \\
\text { Odds ratio (95\% CI) }\end{array}$ & $p$ value & $\begin{array}{c}\text { Multivariate analysis } \\
\text { Odds ratio (95\% CI) }\end{array}$ \\
\hline Age & $1.095(0.503-2.386)$ & 0.819 & $4.132(1.414-12.048)$ \\
Smoking (yes versus no) & $2.488(1.037-5.952)$ & 0.039 & - \\
Systolic BP & $2.013(0.922-4.394)$ & 0.078 & 0.009 \\
Diastolic BP & $1.467(0.677-3.178)$ & 0.330 & - \\
Serum calcium & $0.576(0.264-1.255)$ & 0.164 & $3.487(1.34-9.090)$ \\
Serum phosphate & $2.779(1.256-6.149)$ & 0.011 & - \\
HDL cholesterol & $1.535(0.684-3.444)$ & 0.298 & - \\
LDL cholesterol & $0.433(0.192-0.977)$ & 0.042 & 0.801 \\
Triglyceride & $0.690(0.308-1.546)$ & 0.367 & 0.010 \\
Blood glucose & $0.440(0.198-0.980)$ & 0.043 & - \\
HbAlc & $0.670(0.292-1.534)$ & 0.342 & 0.072 \\
C-reactive protein & $0.369(0.143-0.952)$ & 0.036 & 0.390 \\
AAC grade (versus grade 0) & $1.338(0.4111-4.366)$ & 0.627 & - \\
$\quad$ Grade 1 & $2.141(0.700-6.535)$ & 0.179 & $4.626(1.241-17.244)$ \\
Grade 2 & $4.444(1.353-14.705)$ & 0.012 & $4.333(1.134-16.551)$ \\
Grade 3 & & & 0.023 \\
\hline
\end{tabular}

BP, blood pressure; HbAlc, hemoglobin Alc; HDL, high density lipoprotein; LDL, low density lipoprotein; $\mathrm{rSO}_{2}$, regional saturation of oxygen; AAC, aortic arch calcification; CI, confidence interval.

performed using IBM SPSS Statistics for Windows, version 19.0. Differences with values of $p<0.05$ were considered statistically significant.

\section{Results}

In this study, the mean cerebral $\mathrm{rSO}_{2}$ at rest in patients receiving $\mathrm{HD}$ was $48.6 \pm 9.6 \%$. Patients receiving $\mathrm{HD}$ were divided into four groups by AAC grade (from grade 0 to grade 3 ). There were 23 patients at grade 0,24 patients at grade 1, 30 patients at grade 2 , and 27 patients at grade 3 . Table 1 shows the patients' characteristics in each group. Several factors differed significantly among these four groups: age, HD duration, primary disease causing HD requirement, history of IHD, smoking, albumin, and cerebral $\mathrm{rSO}_{2}$. In particular, as shown in Figure 1, cerebral $\mathrm{rSO}_{2}$ values decreased as $\mathrm{AAC}$ progressed, and these values at AAC grade 3 were significantly lower than those at AAC grades 0 and 1 (AAC grade $0,53.7 \pm 8.3 \%$; grade $1,50.1 \pm 10.1 \%$; grade $2,48.3 \pm 8.6 \%$; grade $3,43.2 \pm 8.8 \%$; AAC grade 3 versus grade $0, p<$ 0.01 ; AAC grade 3 versus grade $1, p<0.05)$. Furthermore, multivariate logistic regression analysis was performed using the following atherosclerotic parameters: smoking, systolic $\mathrm{BP}$, serum calcium, serum phosphate, low density lipoprotein cholesterol, blood glucose, C-reactive protein, and AAC grades 2 and $3\left(p<0.20\right.$ compared with cerebral $\mathrm{rSO}_{2}$ values less than mean monitored in this study in univariate analysis). As shown in Table 2, AAC grade 2 (odds ratio [OR] $=4.626,95 \%$ confidence interval $[\mathrm{CI}]=1.241-17.244)$ and AAC grade $3(\mathrm{OR}=4.333,95 \% \mathrm{CI}=1.134-16.551)$ in addition to serum phosphate $(\mathrm{OR}=3.487,95 \% \mathrm{CI}=1.340-9.090)$ and history of smoking ( $\mathrm{OR}=4.132,95 \% \mathrm{CI}=1.414-12.048)$ were independently associated with cerebral $\mathrm{rSO}_{2}$ values less than the mean in this study.

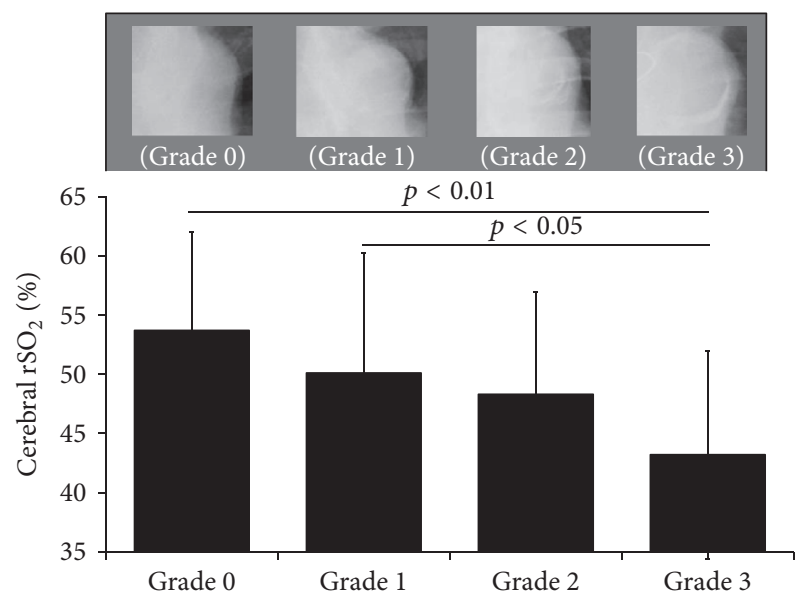

FIGURE 1: Association between AAC grade and cerebral $\mathrm{rSO}_{2}$ value. $\mathrm{AAC}$, aortic arch calcification; $\mathrm{rSO}_{2}$, regional saturation of oxygen.

\section{Discussion}

Previous reports in nondialysis patients have shown that AAC is related to the presence of diabetes mellitus and renal dysfunction [5] and a strong independent predictor of cardiovascular events, apart from the traditional risk factors [8]. On the other hand, in end-stage renal disease patients, vascular calcification, including AAC, is usually accelerated by mineral and bone disorder $[26,27]$ and associated with cardiovascular and all-cause mortality [26, 28-30]. Therefore, it is important to pay attention to the presence of vascular calcification, which is represented by AAC, in the clinical setting. 
However, despite the large number of studies addressing the correlation between AAC and cardiovascular disease, few reports have examined the correlation between AAC and cerebrovascular disorders [7, 31]. Using magnetic resonance imaging on ischemic stroke patients not receiving dialysis, AAC was reportedly associated with cerebral smallartery occlusion and white matter disease [7]; therefore, such vascular disorders might directly influence the cerebral circulation. Furthermore, cerebral circulation dysregulation leads to a decrease in regional cerebral blood flow [32] and leukoaraiosis, an indicator of cerebral ischemia with a high prevalence in the anterior circulation of the brain, has also been reported as an independent risk factor for stroke [33] in dialysis patients. In the present study, decreased cerebral $\mathrm{rSO}_{2}$ values may have reflected a decreased oxygen supply in the brain via cerebral macro- and/or microcirculation impairments, which may explain the reason why AAC progression was negatively associated with cerebral $\mathrm{rSO}_{2}$ value.

The multivariate regression analysis in this study demonstrated that serum phosphate level, history of smoking, and higher AAC grade were independently associated with cerebral $\mathrm{rSO}_{2}$. Hyperphosphatemia impairs endothelial function by increasing reactive oxygen species and inhibiting endothelial nitric oxide synthase [34] and contributes to vascular calcification progression [30], which would lead to poor prognosis in patients receiving $\mathrm{HD}$ [35]. Thus, cerebral $\mathrm{rSO}_{2}$ might be affected by cerebral atherosclerosis progression induced by hyperphosphatemia, although chronic kidney disease -mineral and bone disorder was well-managed in the HD patients in this study. Smoking is a well-known major traditional factor of atherosclerosis. Furthermore, smoking itself reportedly injures endothelial cells $[36,37]$ and accelerates vascular atherosclerotic changes. Thus, this might be why a history of smoking was significantly associated with cerebral oxygenation in this study.

Cerebral $\mathrm{rSO}_{2}$ values were significantly associated with the higher AAC grade, which might indicate that vascular disease with $\mathrm{AAC}$ decreases cerebral $\mathrm{rSO}_{2}$ values. The presence of AAC was associated with arterial stiffness dysregulation [38] and carotid distensibility [39]. Furthermore, arterial stiffness disorders are related to endothelial dysfunction [40] and lead to cerebral circulation dysregulation [41]. Therefore, the presence of AAC might be associated with cerebral microcirculatory impairment via the arterial stiffness disorders, which may have led to the decreased cerebral $\mathrm{rSO}_{2}$ in this study.

This study had several limitations. First, the sample size was relatively small and the most of the patients were men. This study was also cross-sectional; therefore, it remains unclear whether the relationships confirmed in this study reflect causes or effects. Furthermore, AAC was only confirmed on the basis of chest radiography in each patient; therefore, elevated vascular calcification might not be accurate compared with other imaging examinations, including computed tomography and magnetic resonance angiography. Therefore, further studies are needed to clarify the associations between vascular calcification, including $\mathrm{AAC}$, and cerebral oxygenation in patients receiving HD.

\section{Conclusions}

Cerebral $\mathrm{rSO}_{2}$ values significantly decreased as AAC progressed. Furthermore, cerebral $\mathrm{rSO}_{2}$ value was independently associated with a higher AAC grade in addition to serum phosphate level and history of smoking.

\section{Conflicts of Interest}

The authors declare no conflicts of interest.

\section{Authors' Contributions}

Kiyonori Ito and Susumu Ookawara contributed equally to this paper.

\section{Acknowledgments}

The authors thank the study participants and the clinical dialysis center staff in our hospital. This work was supported by a grant from the Japanese Association of Dialysis Physicians (no. 27088) to Susumu Ookawara and a grant from The Kidney Foundation, Japan (JKFB16-3), to Susumu Ookawara.

\section{References}

[1] S. Nakai, N. Hanafusa, I. Masakane, and etal., "An overview of regular dialysis treatment in Japan," Therapeutic Apheresis and Dialysis, vol. 18, no. 6, pp. 535-602, 2014.

[2] S. Sedaghat, M. W. Vernooij, E. Loehrer et al., "Kidney function and cerebral blood flow: the Rotterdam Study, Journal of the American Society of Nephrology, vol. 27, no. 3, pp. 715-721, 2015.

[3] T. Naganuma, J. Uchida, K. Tsuchida et al., "Silent cerebral infarction predicts vascular events in hemodialysis patients," Kidney International, vol. 67, no. 6, pp. 2434-2439, 2005.

[4] H. Shima, E. Ishimura, T. Naganuma et al., "Decreased kidney function is a significant factor associated with silent cerebral infarction and periventricular hyperintensities," Kidney and Blood Pressure Research, vol. 34, no. 6, pp. 430-438, 2011.

[5] H. Hashimoto, K. Iijima, M. Hashimoto et al., "Validity and usefulness of aortic arch calcification in chest X-ray," Journal of Atherosclerosis and Thrombosis, vol. 16, no. 3, pp. 256-264, 2009.

[6] G. Symeonidis, N. Papanas, I. Giannakis, and etal., "Gravity of aortic arch calcification as evaluated in adult Greek patients," International Angiology, vol. 21, no. 3, pp. 233-236, 2002.

[7] Y. Ueno, A. Okuzumi, M. Watanabe et al., "Cerebral small artery diseases may be associated with aortic arch calcification in stroke patients," Journal of Atherosclerosis and Thrombosis, vol. 21, no. 10, pp. 1011-1021, 2014.

[8] K. Iijima, H. Hashimoto, and M. Hashimoto, "Aortic arch calcification detectable on chest X-ray is a strong independent predictor of cardiovascular events beyond traditional risk factors," Atherosclerosis, vol. 210, no. 1, pp. 137-144, 2010.

[9] M. Calderon-Arnulphi, A. Alaraj, S. Amin-Hanjani et al., "Detection of cerebral ischemia in neurovascular surgery using quantitative frequency-domain near-infrared spectroscopy," Journal of Neurosurgery, vol. 106, no. 2, pp. 283-290, 2007.

[10] A. Casati, G. Fanelli, P. Pietropaoli et al., "Continuous monitoring of cerebral oxygen saturation in elderly patients undergoing 
major abdominal surgery minimizes brain exposure to potential hypoxia," Anesthesia and Analgesia, vol. 101, no. 3, pp. 740747, 2005.

[11] J. Malik, J. Kudlicka, J. Lachmanova et al., "Tissue ischemia worsens during hemodialysis in end-stage renal disease patients," Journal of Vascular Access, vol. 18, no. 1, pp. 47-51, 2017.

[12] H. Miyazawa, S. Ookawara, and K. Tabei, "Aggravation of cerebral oxygenation due to intradialytic hypotension induced by blood volume reduction during hemodialysis: a case report," Therapeutic Apheresis and Dialysis, vol. 19, no. 5, pp. 525-527, 2015.

[13] J. M. Murkin, S. J. Adams, R. J. Novick et al., "Monitoring brain oxygen saturation during coronary bypass surgery: a randomized, prospective study," Anesthesia and Analgesia, vol. 104, no. 1, pp. 51-58, 2007.

[14] K. Nishiyama, N. Ito, T. Orita et al., "Regional cerebral oxygen saturation monitoring for predicting interventional outcomes in patients following out-of-hospital cardiac arrest of presumed cardiac cause: a prospective, observational, multicentre study," Resuscitation, vol. 96, pp. 135-141, 2015.

[15] T. Hoshino, S. Ookawara, S. Goto et al., "Evaluation of cerebral oxygenation in patients undergoing long-term hemodialysis," Nephron - Clinical Practice, vol. 126, no. 1, pp. 57-61, 2014.

[16] K. Ito, S. Ookawara, Y. Ueda et al., "Factors affecting cerebral oxygenation in hemodialysis patients: cerebral oxygenation associates with $\mathrm{pH}$, hemodialysis duration, serum albumin concentration, and diabetes mellitus," PLoS ONE, vol. 10, no. 2, Article ID e0117474, 2015.

[17] M. Ferrari, L. Mottola, and V. Quaresima, "Principles, techniques, and limitations of near infrared spectroscopy," Canadian Journal of Applied Physiology, vol. 29, no. 4, pp. 463-487, 2004.

[18] J. D. Tobias, "Cerebral oxygenation monitoring: near-infrared spectroscopy," Expert Review of Medical Devices, vol. 3, no. 2, pp. 235-243, 2006.

[19] P. M. A. Lemmers, M. C. Toet, and F. Van Bel, "Impact of patent ductus arteriosus and subsequent therapy with indomethacin on cerebral oxygenation in preterm infants," Pediatrics, vol. 121, no. 1, pp. 142-147, 2008.

[20] S. Hyttel-Sorensen, L. C. Sorensen, J. Riera, and G. Greisen, "Tissue oximetry: a comparison of mean values of regional tissue saturation, reproducibility and dynamic range of four NIRS-instruments on the human forearm," Biomedical Optics Express, vol. 2, no. 11, pp. 3047-3057, 2011.

[21] J. Schmitz, G. Pichler, B. Schwaberger, B. Urlesberger, N. Baik, and C. Binder, "Feasibility of long-term cerebral and peripheral regional tissue oxygen saturation measurements," Physiological Measurement, vol. 35, no. 7, pp. 1349-1355, 2014.

[22] K. Hongo, S. Kobayashi, H. Okudera, M. Hokama, and F. Nakagawa, "Noninvasive cerebral optical spectroscopy: depthresolved measurements of cerebral haemodynamics using indocyanine green," Neurological Research, vol. 17, no. 2, pp. 89-93, 1995.

[23] H. Maslehaty, U. Krause-Titz, A. K. Petridis, H. Barth, and H. M. Mehdorn, "Continuous measurement of cerebral oxygenation with near-infrared spectroscopy after spontaneous subarachnoid hemorrhage," ISRN Neurol, Article ID 907187, 2012.

[24] N. X. Chen and S. M. Moe, "Vascular calcification: pathophysiology and risk factors," Current Hypertension Reports, vol. 14, no. 3, pp. 228-237, 2012.

[25] F. J. Alenghat, "The prevalence of atherosclerosis in those with inflammatory connective tissue disease by race, age, and traditional risk factors," Scientific Reports, vol. 6, Article ID 20303, 2016.

[26] G. M. London, A. P. Guérin, S. J. Marchais, F. Métivier, B. Pannier, and H. Adda, "Arterial media calcification in end-stage renal disease: impact on all-cause and cardiovascular mortality," Nephrology Dialysis Transplantation, vol. 18, no. 9, pp. 1731-1740, 2003.

[27] C. M. Shanahan, M. H. Crouthamel, A. Kapustin, and C. M. Giachelli, "Arterial calcification in chronic kidney disease: key roles for calcium and phosphate," Circulation Research, vol. 109, no. 6, pp. 697-711, 2011.

[28] J. A. Abdelmalek, P. Stark, C. P. Walther, J. H. Ix, and D. E. Rifkin, "Associations between coronary calcification on chest radiographs and mortality in hemodialysis patients," American Journal of Kidney Diseases, vol. 60, no. 6, pp. 990-997, 2012.

[29] M. Komatsu, M. Okazaki, K. Tsuchiya, H. Kawaguchi, and K. Nitta, "Aortic arch calcification predicts cardiovascular and allcause mortality in maintenance hemodialysis patients," Kidney and Blood Pressure Research, vol. 39, no. 6, pp. 658-667, 2014.

[30] M. Noordzij, E. M. Cranenburg, L. F. Engelsman et al., "Progression of aortic calcification is associated with disorders of mineral metabolism and mortality in chronic dialysis patients," Nephrology Dialysis Transplantation, vol. 26, no. 5, pp. 16621669, 2011.

[31] Y. Shimada, Y. Ueno, Y. Tanaka et al., "Aging, aortic arch calcification, and multiple brain infarcts are associated with aortogenic brain embolism," Cerebrovascular Diseases, vol. 35, no. 3, pp. 282-290, 2013.

[32] H. Kanai, H. Hirakata, H. Nakane et al., "Depressed cerebral oxygen metabolism in patients with chronic renal failure: a positron emission tomography study," American Journal of Kidney Diseases, vol. 38, 1, no. 4, pp. S129-S133, 2001.

[33] D. Inzitari, "Leukoaraiosis: an independent risk factor for stroke?" Stroke, vol. 34, no. 8, pp. 2067-2071, 2003.

[34] T. V. Van, E. Watari, Y. Taketani et al., "Dietary phosphate restriction ameliorates endothelial dysfunction in adenineinduced kidney disease rats," Journal of Clinical Biochemistry and Nutrition, vol. 51, no. 1, pp. 27-32, 2012.

[35] G. M. London, "Cardiovascular calcifications in uremic patients: clinical impact on cardiovascular function," Journal of the American Society of Nephrology, vol. 14, pp. 305-309, 2003.

[36] K. Iseki, "Factors influencing the development of end-stage renal disease," Clinical and Experimental Nephrology, vol. 9, no. 1, pp. 5-14, 2005.

[37] S. R. Orth, E. Ritz, and R. W. Schrier, "The renal risks of smoking," Kidney International, vol. 51, no. 6, pp. 1669-1677, 1997.

[38] A. Sekikawa, C. Shin, J. D. Curb et al., "Aortic stiffness and calcification in men in a population-based international study," Atherosclerosis, vol. 222, no. 2, pp. 473-477, 2012.

[39] M. J. Blaha, M. J. Budoff, J. J. Rivera et al., "Relationship of carotid distensibility and thoracic aorta calcification: multiethnic study of atherosclerosis," Hypertension, vol. 54, no. 6, pp. 1408-1415, 2009.

[40] K. Yufu, N. Takahashi, M. Hara, T. Saikawa, and H. Yoshimatsu, "Measurement of the brachial-ankle pulse wave velocity and flow-mediated dilatation in young, healthy smokers," Hypertension Research, vol. 30, no. 7, pp. 607-612, 2007.

[41] R. Dubin, C. Owens, W. Gasper, P. Ganz, and K. Johansen, "Associations of endothelial dysfunction and arterial stiffness with intradialytic hypotension and hypertension," Hemodialysis International, vol. 15, no. 3, pp. 350-358, 2011. 


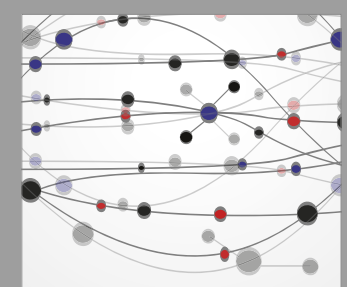

The Scientific World Journal
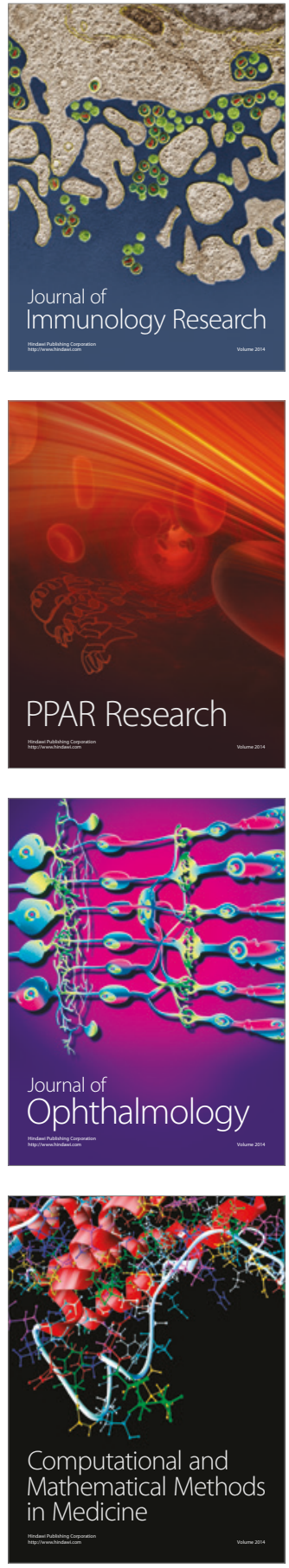

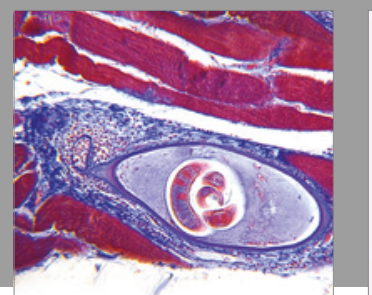

Gastroenterology Research and Practice
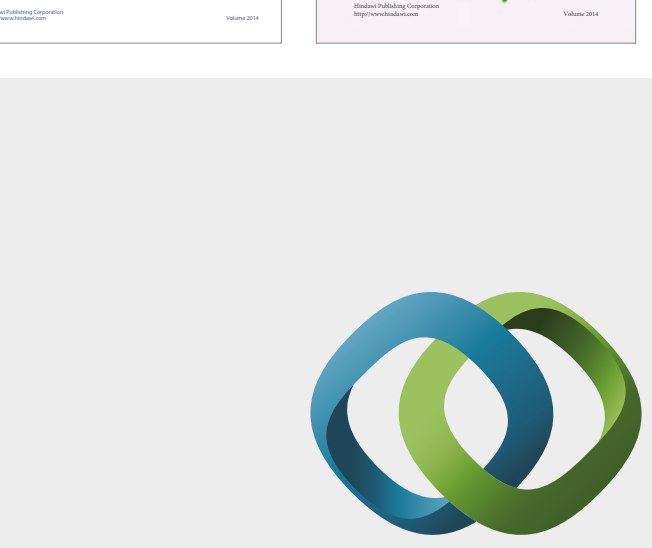

\section{Hindawi}

Submit your manuscripts at

https://www.hindawi.com

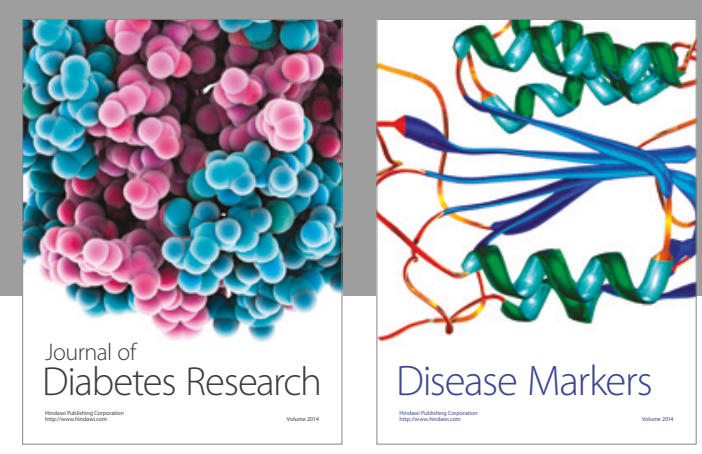

Disease Markers
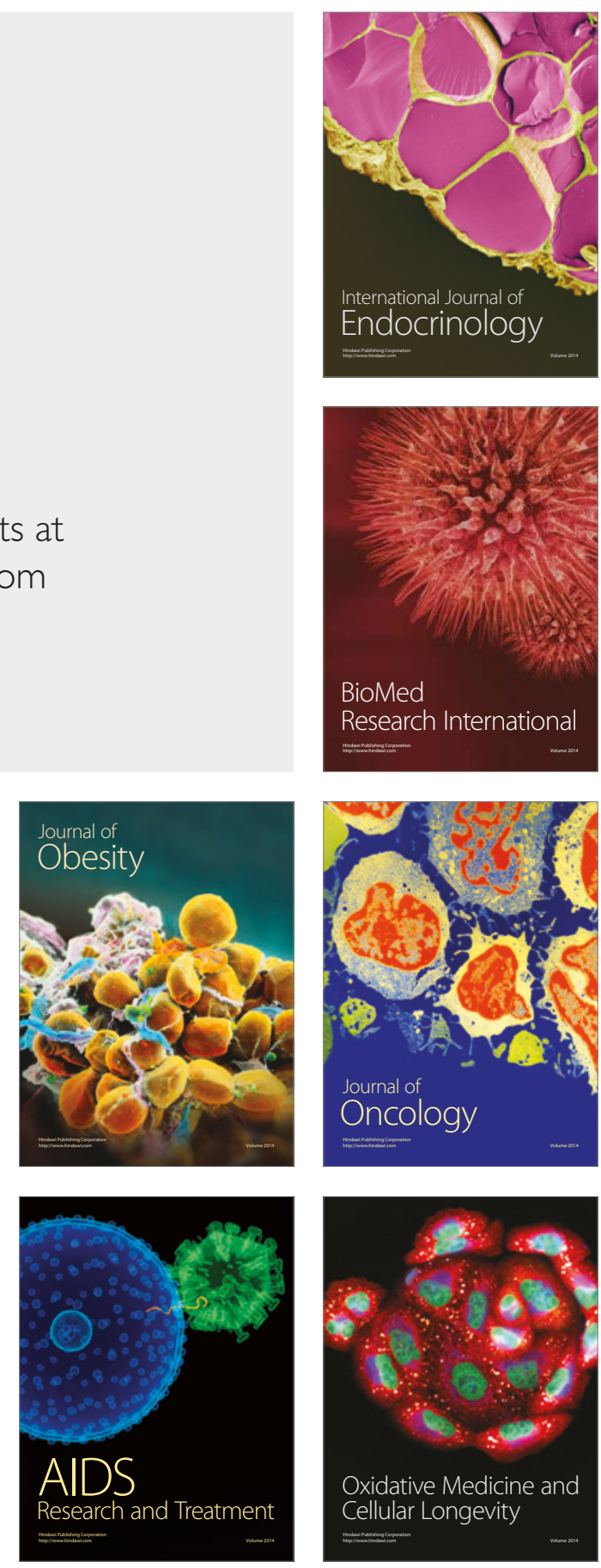